\title{
Displasia folicular de la capa negra canina
}

\author{
Follicular dysplasia of the canine black coat
}

\author{
Yasmir Arroyo M ${ }^{1,2}$ MVZ; Luis Carlos Hincapié $\mathrm{G}^{1,3} \mathrm{MVZ}$ \\ ${ }^{1}$ Universidad de Córdoba, Facultad de Medicina Veterinaria y Zootecnia, \\ Departamento de Ciencias Pecuarias, Montería, Córdoba, Colombia. \\ ${ }^{2}$ Miembro de la Sociedad Latinoamericana De Dermatología Veterinaria \\ ${ }^{3}$ Universidad de Córdoba, Facultad de Medicina Veterinaria y Zootecnia, \\ Grupo de Investigación en Producción Animal Tropical, U Colombia.
}

\section{KEYWORDS:}

Hair follicle; hypotrocis; dysplasia; melanin.

\section{PALABRAS CLAVE:}

Folículo piloso; hipotrocis; displasia; melanina.

\section{ABSTRACT}

Follicular dysplasia of the black layer, is a dermatopathy restricted to areas of black hair of dogs with two or more layers of colors. Studies have mentioned a primary problem in the hair follicle, where an autosomal recessive inheritance is involved. The diagnosis is made thanks to the clinical history, physical examination, tricogram and histopathological examination of the affected area. Case report. A mongrel canine with short hair, a 2-year-old male with a two-color coat, presents with progressive hair loss in the black areas from the month and a half of age. The general clinical examination did not show any physiological alteration, getting as relevant finding the presence of circumscribed alopecia and hypotricosis in the black areas located in the temporal zone of the head and the ears, apruriginosa, with mild seborrhea. Skin scraping is performed, this being negative for scabies mites, and subsequently sending a sample for histopathology with the report of follicular dysplasia of black hair. Conclusion. The follicular dysplasia of the black hair of the canine is a dermatological condition of little presentation in the veterinary clinic. So, reports like this, allow to demonstrate the presence of the pathology, and with that, to increase the knowledge of this type of dermatopathy.

\section{RESUMEN}

La displasia folicular de la capa negra, es una dermatopatía restringida a las zonas de pelo negro de perros con dos o más capas de colores. Estudios mencionan haber un problema primario en el folículo piloso, donde está involucrada una herencia autosómica recesiva. El diagnóstico se hace gracias a la historia clínica, examen físico, tricograma y examen histopatológico del área afectada. Reporte del caso. Un canino mestizo de pelo corto, macho entero de 2 años de edad, de pelaje bicolor, se presenta con caída progresiva del pelo en las áreas negras desde el mes y medio de edad. Al examen clínico general no evidenció ninguna alteración fisiológica, encontrándose como hallazgo relevante la presencia de alopecia circunscrita e hipotricosis en las áreas negras ubicadas en la zona temporal de la cabeza y las orejas, apruriginosa, con leve seborrea. Se procede a realizar raspado de piel, siendo éste negativo para ácaros de la sarna, y a enviar posteriormente muestra para histopatología con el reporte de displasia folicular del pelo negro. Conclusión. La displasia folicular del pelo negro del canino es una afección dermatológica de escasa presentación en la clínica veterinaria. Por lo que reportes como éste, permiten demostrar la presencia de la patología, y con ello, aumentar el conocimiento de este tipo de dermatopatía.
INFORMACIÓN

Recibido: 02-03-2018;

Publicado: 02-07-2018

Correspondencia autor: mascotasclinica@gmail.com 


\section{INTRODUCCIÓN.}

Las displasias foliculares son dermatopatías de origen hereditario, en las que se produce un anormal desarrollo anatómico y de melanización de unidades pilosebáceas (SEABRA et al., 2015), constituyendo en la clínica diaria veterinaria trastornos patológicos infrecuentes y de patogenia poco conocida, que afecta principalmente a ciertas razas, evidencian clínicamente alteración de la calidad del pelo y evolucionan con alopecia progresiva (RÍOS et al., 1996).

Las displasias foliculares ligadas al color del pelo, también denominadas como displasias cutáneas neuroectodérmicas hereditarias (MECKLENBURG, 2006), son dermatopatías histológica y genéticamente parecidas, y probablemente tratadas similarmente, pero son de aspecto clínico diferente en caninos (RODRIGUES et al., 2007), y dentro de las cuales podemos encontrar: alopecia por dilución de color, donde hallamos la forma difusa y la displasia folicular de la capa negra, la cual es la forma localizada (SEABRA et al., 2015).

La displasia folicular de la capa negra canina (BHFD, por sus siglas en ingles), es una dermatopatía rara restringida a las zonas de la capa negra de perros con dos o más capas de colores (BOMHARD et al., 2006). Estudios mencionan haber un problema primario en el folículo piloso, donde está involucrada una herencia autosómica recesiva (SEABRA et al., 2015), lo que conduce a una producción anómala de los melanocitos sobre los folículos pilosos displásicos, provocando un aglomerado de melanina en los bulbos foliculares y de melanosomas en estadio IV en los melanocitos de la epidermis, con la insuficiente transferencia de melanina hacia los queratinocitos adyacentes (BOMHARD et al., 2006, SCHMUTZ et al., 1998). Los caninos afectados por este tipo de patologías, son perros jóvenes, con manto de dos o más colores y uno de ellos de color negro, que nacen normales y van evidenciando cambios como la pérdida del brillo del pelo negro a partir del mes de edad de vida y pérdida progresiva del pelo en las áreas negras de pelo, hasta quedar sin pelo alrededor de los 6 y 9 meses de edad, respetando las áreas de pelo de color blanco (RODRIGUES et al., 2007, SAYURI et al., 2012). No hay predilección sexual (SEABRA et al., 2015).

La displasia folicular de los pelos negros en caninos afecta a perros mestizos, como a caninos de diferentes razas como la Border Collie, Jack Russel Terrier, Salukis, Basset Hound, Dachshund, Yorkshire Terrier, Beagle, Doberman, Cocker Spaniel, Pointer, Papillon y Terrier Brasilero (SEABRA et al., 2015, SCHMUTZ et al., 1998, SAYURI et al., 2012, CUNHA et al., 2005). Es característica la alopecia progresiva en las áreas de pelo negro, que puede estar acompañado de descamación, piel seca, pelos quebradizos y sin brillo, contribuyendo a crear el ambiente para la infección secundaria bacteriana recurrente (SEABRA et al., 2015, RODRIGUES et al., 2007, BOMHARD et al., 2006). Las principales áreas corporales afectadas de los perros son la cabeza, orejas, cuello y tronco (RODRIGUES et al., 2007).

El diagnóstico de esta enfermedad se hace en base a una minuciosa y estricta historia clínica, examen físico, tricograma y examen histopatológico del área afectada (LOPEZ et al., 2013). No existe tratamiento efectivo para esta patología, pudiéndose administrar melatonina en dosis de 3-6 mg/animal cada 12 horas como tratamiento tentativo (SEABRA et al., 2015). El pronóstico de la displasia folicular de la capa negra canina es favorable, la alopecia es irreversible, dificultando la parte estética del canino, sin afectar la salud y calidad de vida del animal (PALUMBO et al., 2012). Este tipos de mascotas no se recomienda reproducir, aconsejándose esterilizarlos para prevenir la transmisión de los genes que ayudan en el desarrollo de la enfermedad, al ser de carácter hereditario (CUNHA et al., 2005).

En Colombia, no se encuentra reportes en la literatura de casos de displasia folicular de la capa negra canina, solo conociendo casos anecdóticos. En el presente trabajo, se describe un caso en un canino mestizo. 


\section{Reporte del caso.}

Un canino mestizo de pelo corto, macho entero de 2 años de edad, de pelaje bicolor (blanco y negro), sin vacunas y desparasitación vigente, alimentado con concentrado comercial, con peso de $8 \mathrm{~kg}$, fue atendido en el servicio de dermatología de Mascotas Clínica Veterinaria Sincelejo el día 15 de marzo de 2017.

Dentro de los antecedentes que informó la propietaria se encuentra la caída progresiva del pelo en las áreas negras desde el mes y medio de edad, por lo que fue llevado a otras clínicas veterinarias, donde se controlaba momentáneamente la seborrea y el mal olor al finalizar los diferentes tratamientos instaurados, pero sin evidenciar el crecimiento de nuevos pelos negros de las áreas alopécicas. El perro reside en un apartamento, en el municipio de Sincelejo, Sucre, junto a otros caninos provenientes de otros hogares, totalmente sanos y sin presencia de alguna alteración dermatológica. Se desconoce la procedencia y ubicación de sus progenitores, y el destino de sus hermanos.

Al examen clínico general presenta frecuencia cardiaca de $88 \mathrm{lpm}$, frecuencia respiratoria de $24 \mathrm{rpm}$, temperatura de $38.7^{\circ} \mathrm{C}$, buena condición corporal (4/5), tiempo de llenado capilar 2 segundos, pulso rítmico y fuerte, sonidos normales a la auscultación pulmonar y cardiaca, reflejo tusígeno negativo, mucosas rosadas, actitud alerta, demostrando que al examen físico no evidenció ninguna alteración sistémica, encontrándose como hallazgo relevante la presencia de alopecia circunscrita e hipotricosis en las áreas negras ubicadas en la zona temporal de la cabeza y las orejas, apruriginosa, con leve seborrea (Figura 1), sin presencia de otras lesiones dermatológicas y sin antecedentes de otras enfermedades.

Se procede a realizar raspado de piel, siendo éste negativo para ácaros de la sarna, y a enviar posteriormente muestra para histopatología de las áreas negras sin pelo afectadas, con el siguiente reporte: Se observa una epidermis con un epitelio

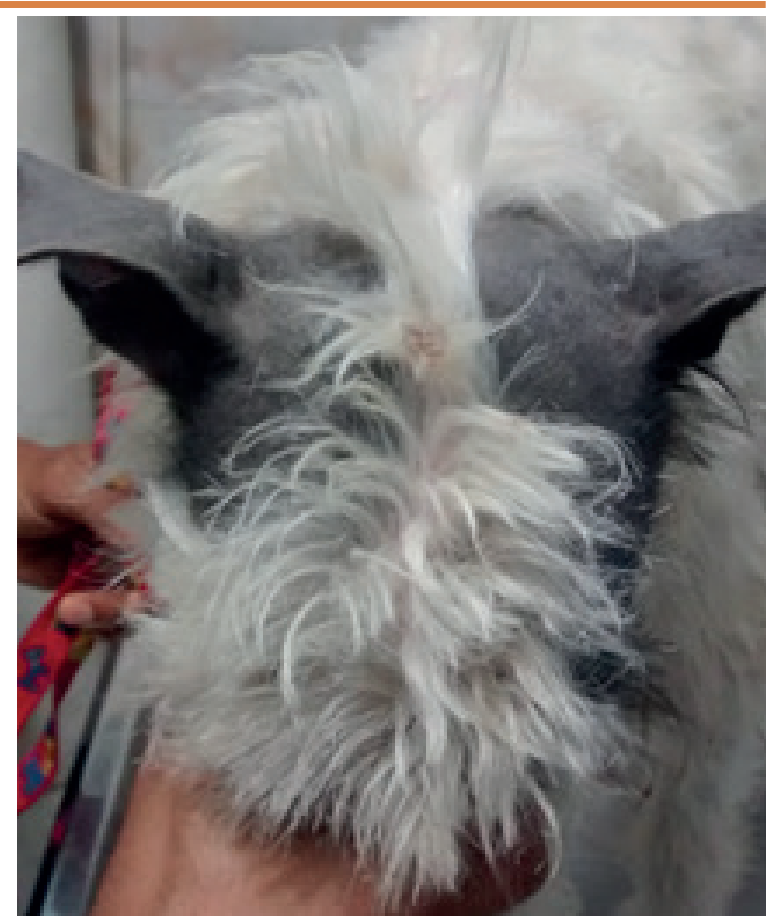

Figura 1. Alopecia circunscrita en áreas negras de pelo.

estratificado plano con hiperqueratosis leve y focos múltiples con pigmentación de melanina en la capa basal y la lámina propia. A nivel subepitelial se encuentra atrofia de las glándulas sebáceas, algunos folículos pilosos dilatados y con presencia de queratina en su interior. En zonas cercanas, los folículos pilosos presentan poco desarrollo y con presencia de melanina en su interior así como en la periferia en cantidad variable y en el bulbo del folículo (Incontinencia pigmentaria perifolicular) (Figura 2). Focos pequeños de melanina también está presente en el tejido adiposo adyacente. No se observa reacción de polimorfo-nucleares ni de células linfoplasmacitarias a nivel perivascular. El edema observado es escaso. Diagnóstico histopatológico: Hallazgos compatibles con una displasia folicular del pelo negro.

Se consideró como diagnóstico definitivo la displasia folicular del pelo negro debido a los datos encontrados en la anamnesis, las características propias de la patología encontradas al examen clínico general y al resultado emitido en la histopatología.

Se le instauró un tratamiento con baños de champú a base de clorhexidina al 3\% cada 


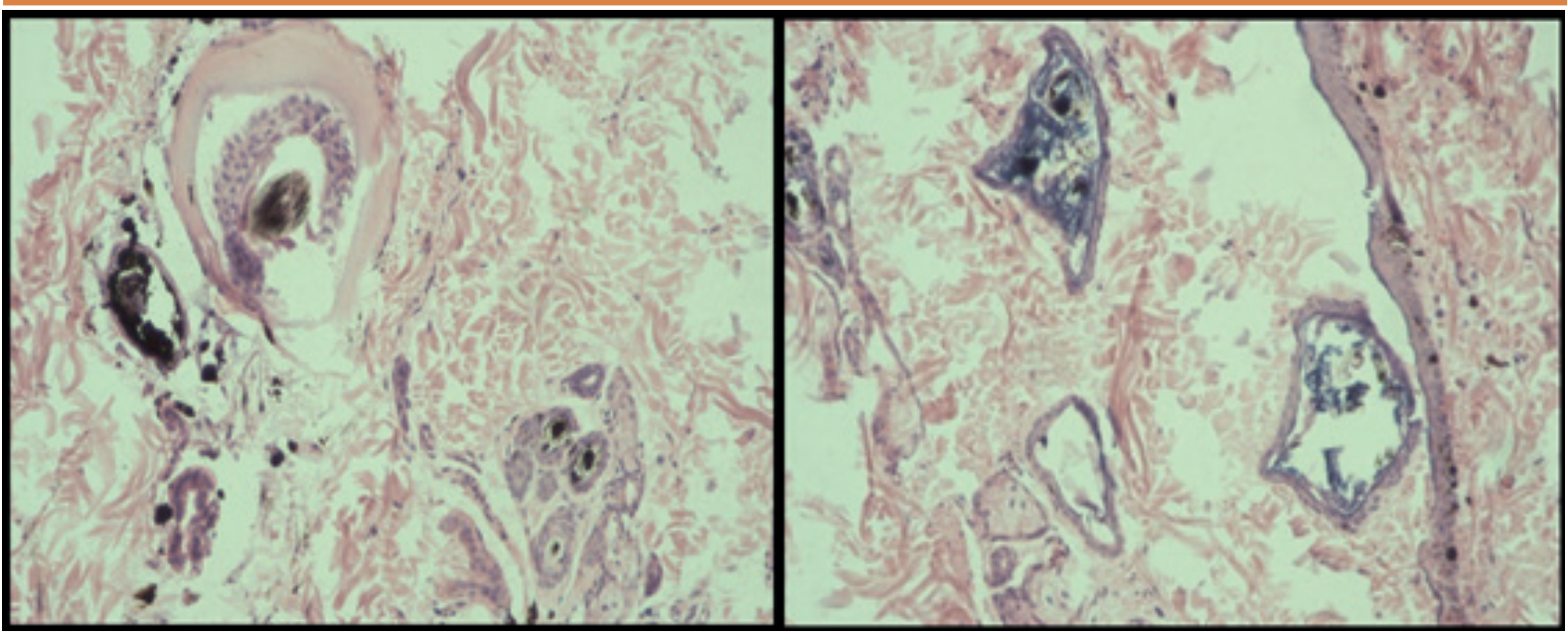

Figura 2. Hallazgos histopatológicos compatibles con la displasia folicular de la capa negra.

3 días por 30 días, suplemento alimenticio a base de omegas y vitamina E. Conociendo de antemano, que la displasia folicular del pelo negro no tiene tratamiento efectivo, solo siendo un problema cosmético para el perro, sin provocar lesión alguna en la salud del animal, y solo pudiéndose controlar las afecciones secundarias.

Debido al carácter hereditario de la displasia folicular del pelo negro, se le orientó a la propietaria evitar la decisión de reproducir a su mascota, recomendándole realizar la orquiectomía total del paciente, para así evitar la propagación de ésta dermatopatía.

\section{DISCUSIÓN}

La patogenia de este tipo de dermatopatías displásicas es confusa, y aún no se ha descubierto a ciencia cierta el mecanismo fisiopatológico por el cual se desarrollan, pero sin duda alguna se tiene claro que proceden de una anomalía primitiva en el folículo piloso, de origen genético (RODRIGUES et al., 2007). Las displasias foliculares asociadas al color comúnmente son debidas a la acumulación de cierta cantidad de melanosomas en estadio IV en los melanocitos ubicados en la epidermis y bulbo piloso, acompañado de nula o insuficiente transferencia hacia los queratinocitos contiguos (BOMHARD et al., 2006, SCHMUTZ et al., 1998, SAYURI et al., 2012). Los cambios histológicos y ultraestructurales de la displasia folicular del pelo negro del canino comparten características con el síndrome de griscelli en el humano (GRISCELLI et al., 1978, MENASCHE et al., 2003). Debido a lo anterior, se plantea un defecto de transporte melanosómico dentro de los melanocitos con modificación de la unidad pigmentaria como parte fundamental del mecanismo patogénico en la displasia folicular (BOMHARD et al., 2006).

Las características clínicas evidenciadas en el presente caso clínico en cuanto a la edad de presentación del inicio de la caída de pelo de las áreas negras, respetando el manto de pelo blanco, al igual que la zona del cuerpo del animal afectado, en éste caso la zona temporal de la cabeza y las orejas, fueron también halladas por Kim S, et al., (KIM et al., 2005), Song T, et al., (SONG et al., 2008), y Knottenbelt y Knottenbelt (KNOTTENBELT y KNOTTENBELT, 1996), en sus respectivos trabajos; siendo éstas las áreas corporales (RODRIGUES et al., 2007), la edad (RODRIGUES et al., 2007, SAYURI et al., 2012), y el grupo racial (SEABRA et al., 2015, SCHMUTZ et al., 1998, SAYURI et al., 2012, CUNHA et al., 2005), que se afectan principalmente.

El diagnóstico del presente cuadro clínico se estableció gracias a una escrupulosa y juiciosa historia clínica, un examen físico ordenado y completo, y el resultado del examen histopatológico del área afectada, que reveló los cambios micromorfologicos 
compatibles con la displasia folicular del pelo negro, como lo describe Lopez T, et al., (LOPEZ et al., 2013). No fue necesaria la realización de exámenes de laboratorio para descartar desordenes endocrinológicos que pueden causar displasia folicular secundaria, debido a las característica clínicas e histopatológicas particulares de la displasia folicular de la capa negra canina (SEABRA et al., 2015). Cabe destacar que el resultado del examen histopatológico del área afectada, evidencia algunas características particulares, pero pueden ser semejantes a las otras displasias foliculares o ciertas endocrinopatías que afectan la piel (RODRIGUES et al., 2007), siendo el aspecto al examen clínico y la estricta anamnesis las que puedan determinar el éxito del diagnóstico.

Hasta este momento no existe un tratamiento específico para este tipo de dermatopatías, instaurándose una terapia exclusivamente regida a reducir la posible infección secundaria y descamación, pudiéndose utilizar champús queratomoduladores, antisépticos y emolientes. Igualmente se puede utilizar un suplemento de ácidos grasos esenciales, para ayudar a mantener o mejorar la película de lípidos de la piel y el aspecto del pelo que persiste en el canino (RODRIGUES et al., 2007).
Es de vital importancia tener presente que algunas patologías que provocan modificación de color y caída permanente de áreas de pelo, además de provocar cambios estéticos, pueden conllevar a procesos patológicos más graves como la dermatitis actínica y carcinomas (PALUMBO et al., 2012).

\section{Conclusión}

La displasia folicular del pelo negro del canino es una afección dermatológica de escasa presentación en la práctica diaria de la clínica veterinaria. De allí la importancia del reporte de casos esporádicos como éste, debido a que permiten demostrar la presencia de la patología, y con ello conlleva a aumentar el conocimiento acerca de las lesiones cutáneas que puede producir este tipo de dermatopatía.

Se destaca la importancia de los diagnósticos diferenciales en casos de pérdida de pelo en áreas focales y ausencia de prurito. Además del gran valor diagnostico que posee una puntual y reflexiva historia clínica, un examen físico sistemático y completo, complementado por el resultado del examen histopatológico del área afectada, apoyando éste último en lo que se había propuesto al momento del examen clínico general.

\section{REFERENCIAS}

Bomhard, W., Mauldin, E., Schmutz, S., Leebs, T., \& Casal, M. (2006). Black hair follicular dysplasia in Large Münsterländer dogs: clinical, histological and ultrastructural features. Veterinary Dermatology, 17: 182-188.

Cunha, F., Silveira, L., Perrone, E., Ferrigno, C., \& Futema, F. (2005). Displasia folicular dos pelos negros. Relato de caso. Braz. J. vet. Res. Anim. Sci., 40: 169.

Griscelli, C., Durandy, A., Guy-Grand, D., Daguillard, F., Herzog, C., \& Prunieras, M. (1978). A syndrome associating partial albinism and immunodeficiency. American Journal of Medicine, 65:691-702.

Kim, S., Kim, Y., Seo, J., Park, J., Jeong, A., Lee, K., \& Oh, T. (2005). Black Hair Follicular Dysplasia in a Shih Tzu. J Vet Clin, 22: 157-159.

Knottenbelt, C., \& Knottenbelt, M. (1996). Black hair follicular dysplasia in a tricolour Jack Russell terrier. Veterinary record, 138: 475-476. 
Lopez, T., de Vargas, S., Nobre, M., Tortato, N., Souza, P., \& Da Silva, G. (2013). Displasia follicular do pêlo preto em canino. Archives of Veterinary Science, 18: 606-608.

Mecklenburg, L. (2006). An overview on congenital alopecia in domestic animals. Veterinary Dermatology, 17: 393-410.

Menasche, G., Hsuan, H., Sanal, O., Feldmann, J., Tezcan, I., Ersoy, F., Houdusse, A., Fischer, A., \& de Saint, G. (2003). Griscelli syndrome restricted to hypopigmentation results from a melanophilin defect (GS3) or a MYO5A F-exon deletion (GS1). J Clin Invest, 112: 450-456.

Palumbo, M., Fabris, V., \& Machado, L. (2012). Carcinoma de células escamosas em um cão com alopecia por diluição de cor. Vet. e Zootec., 19: 507-512.

Ríos, A., Fernández, T., \& Gómez, L. (1996). Displasia folicular en un dobermann adulto de capa negra. Un caso clínico. Clínica Veterinaria de Pequeños Animales, 16: 40-44.

Rodrigues R, da Silva M, Aguiar J, Spanamberg, A., Passos, S., Conceição, E., \& Driemeier, D. (2007). Displasias foliculares ligadas à cor da pelagem em cães: displasia folicular dos pêlos pretos e alopecia por diluição da cor. Acta Scientiae Veterinariae, 35: 119-124.

Sayuri, L., Naves, D., \& Pimenta, A. (2012). Black hair follicular dysplasia in a Brazilian Terrier: a case report. R. bras. Ci. Vet., 19:7-12.

Schmutz, S., Moker, J., Clark, E., \& Shewfelt, R. (1998). Black hair follicular dysplasia: an autossomal recessive condition in dogs. Canadian Veterinary Journal, 39: 644-646.

Seabra, T., Machado, R., Seabra, F., Rosolem, S., \& Franco, V. (2015). Displasia folicular do pelo preto em canino. Acta Veterinaria Brasilica, 9: 284-288.

Song, T., Huang, H., Chang, P., \& Lien, Y. (2008). Canine Black Hair Follicular Dysplasia in a Chihuahua: A Case Report. Journal of Veterinary Clinical Sciences, 1: 21-23. 